\title{
Preparation of Modified Acetate Fiber with Sodium Hydroxide
}

\author{
Haiyan Zhang ${ }^{1, \text { a }}$ \\ ${ }^{1}$ School of Textile \& Garments , Hebei University of Science \& Technology , \\ Shijiazhuang, Hebei, China \\ azhanghaiyan5858@sina.com
}

Keywords: Sodium hydroxide, Acetate fiber, Modified, Fixation rate, Breaking strength, Moisture regain

Abstract. Acetate fiber has many advantages, such as soft silk feel, after dyed bright color, beautiful, weather resistant, good drapability etc. In recent years, acetate fiber has become one of the man-made fiber industry developed rapidly. The deeply researches on the preparation of modified acetate fiber and the performance were carried out. The modified process was as follows: concentration of sodium hydroxide $6 \mathrm{~g} / \mathrm{L}$, time $80 \mathrm{~min}$, room temperature, ratio of water and acetate fiber was 100:1. The hydrophilicity of acetate fiber is improved and the breaking strength is indecreased.

\section{Introduction}

Acetate fiber is a derivative of cellulose fibers. In 1904 the German Bayer company invented dry spinning cellulose acetate fibers. Patents were applied in France and the United Kingdom. The first product of acetate fiber was debuted for more than one hundred years of history. Acetate fiber has a natural silk like soft feel and good at its bright color, beautiful appearance, light fastness, drape pendency, and so on. In recent years, acetate fiber has become one of the man-made fiber industry developed rapidly. [1,2] However, because of the lack of hydrophilic hydroxyl groups in the molecule of the acetate fiber the use of the fiber fabric is limited. How to improve the hydrophilicity of acetate fiber, and improve the comfort of their comfort have become the hot topics.

\section{Theories and Solutions}

A brief introduction on acetate fiber. Acetate is derived from cellulose by reacting purified cellulose from wood pulp with acetic acid and acetic anhydride in the presence of sulfuric acid. It is then put through a controlled, partial hydrolysis to remove the sulfate and a sufficient number of acetate groups to give the product the desired properties. The anhydroglucose unit, is the fundamental repeating structure of cellulose, has three hydroxyl groups which can react to form acetate esters. The most common form of cellulose acetate fiber has an acetate group on approximately two of every three hydroxyls. This cellulose diacetate is known as secondary acetate. Cellulose acetate is dissolved in acetone for extrusion. As the filaments emerge from the spinneret, the solvent is evaporated in warm air, producing fine filaments of cellulose acetate. [3] The structural formulas of acetate fiber are as follows: 

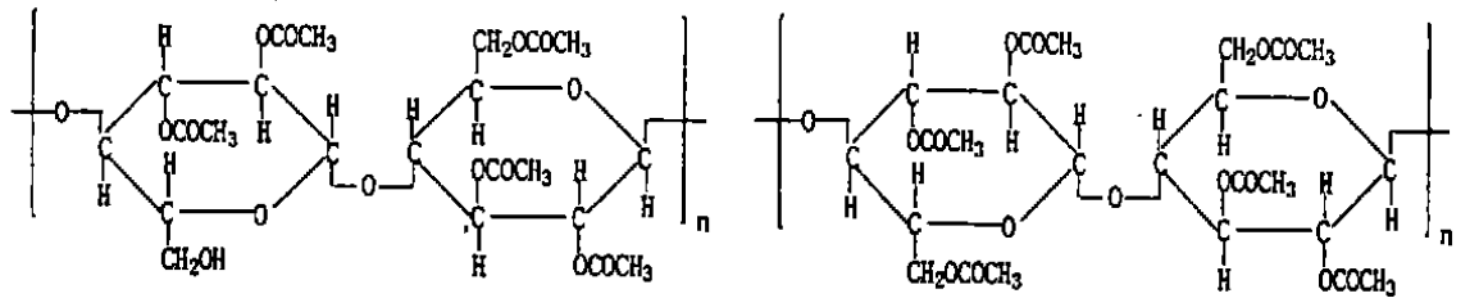

The principle of modified acetate fiber with sodium hydroxide. The hydroxyl on cellulose can react with acetic acid dehydration, the ester bonds is obtained and the hydroxyl groups were closed. In order to obtain a good fiber properties, the hydroxyls were exposed by hydrolysis of part of ester bond. It can not only keep the properties of the original acetate fiber but also can make it hydrophilic. The ester bond is easy to hydrolysis under alkaline conditions, the reaction equation of alkaline hydrolysis as follows:<smiles>CC(=O)OC(=O)C(=O)O[Hg]O</smiles>

Testing solution. After modification of the acetate fiber part of the hydroxyls were exposed and it can be dyed with the reactive dyes. [4,5] So dyeing method with reactive dyes can be used to test the modification effect. The reaction mechanism is as follows:

$$
\text { Cell }-\mathrm{OH} \stackrel{\mathrm{OH}^{-}}{\longrightarrow} \mathrm{CellO}
$$

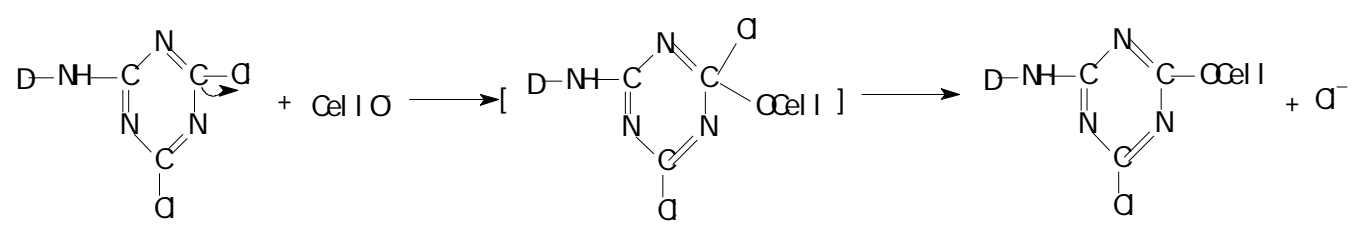

The weight and the breaking strength, moisture regain of the acetate fiber are changed. The weight change can be characterized by mass loss rates.

$$
\text { Mass loss rates }=\frac{\text { weights of acetate fiber }- \text { weights of modified acetate fiber }}{\text { weights of acetate fiber }} * 100 \%
$$

\section{Results and discussions}

The optimum modified technology of acetate fiber. The method for surface modification of acetate fiber was determined through single factor experiment. At room temperature weighing out certain acetate fiber as sample and put it into sodium hydroxide solution for a period of time. The ratio of water and acetate fiber is 100:1. The influence results of concentrations of sodium hydroxide and reaction time on fixation rates with reactive dyes are shown in Fig.1, Fig.2. Fig.1 illustrates when the concentration of sodium hydroxide is $6 \mathrm{~g} / \mathrm{L}$ the fixation rate is maximum and the optimum sodium hydroxide solution concentration at $6 \mathrm{~g} / \mathrm{L}$. Fig. 2 illustrates when the reaction time is $80 \mathrm{~min}$ the fixation rate is maximum and the optimum time is $80 \mathrm{~min}$. The optimum process was as 
follows: sodium hydroxide $6 \mathrm{~g} / \mathrm{L}$, reaction $40 \mathrm{~min}$ at room temperature, ratio of water and cotton fabric was 100:1.

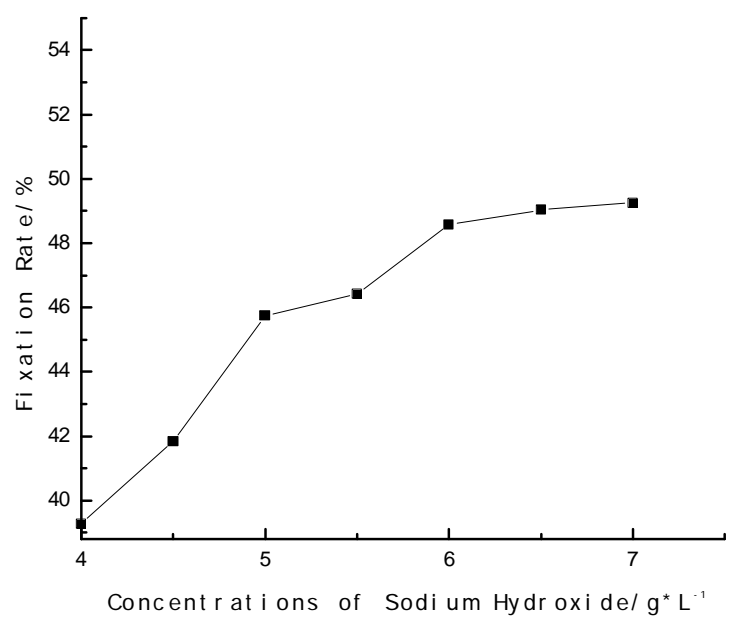

Fig. 1 Relationship between concentrations of sodium hydroxide and fixation rate

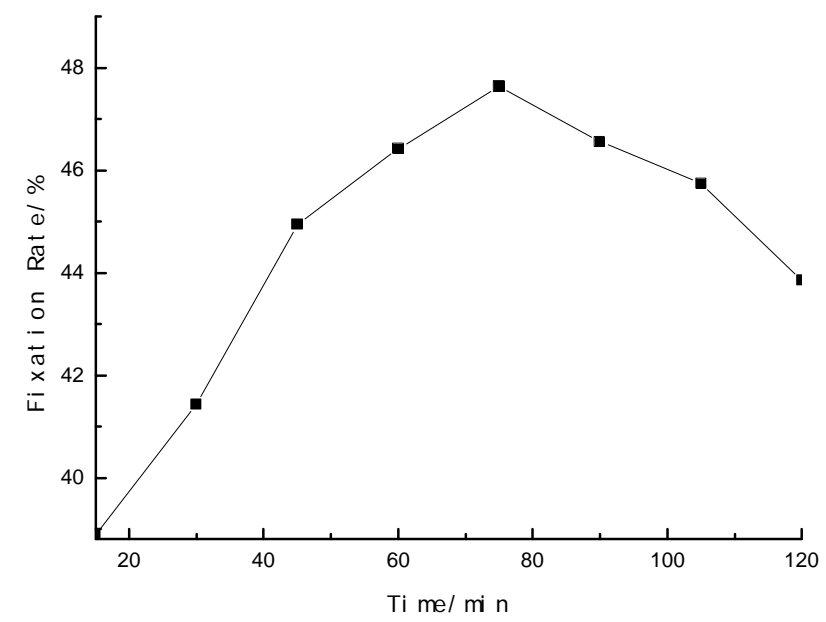

Fig. 2 Relationship between reaction time and fixation rate

The breaking strength, mass loss rates and moisture regain of modified acetate fiber with sodium hydroxide. The breaking strength of modified acetate fiber with sodium hydroxide is changed, it can be seen from the Table 1. Compared with original fabric, the breaking strength of modified acetate fiber is decreased from $10.7 \mathrm{cN} /$ Tex to $9.74 \mathrm{cN} /$ Tex and the moisture regain increased from $6.67 \%$ to $7.38 \%$. Mass loss rates of modified acetate fiber is $23.4 \%$ and the mass loss rates is directly proportional to the amount of sodium hydroxide. 
Table 1 The performance of modified acetate fiber

\begin{tabular}{lccc}
\hline sample & $\begin{array}{c}\text { breaking strength / } \\
\mathrm{cN} \cdot \mathrm{Tex}^{-1}\end{array}$ & mass loss rates/\% & moisture regain/\% \\
\hline acetate fiber & 10.7 & 0 & 6.67 \\
modified acetate fiber & 9.74 & 23.41 & 7.38 \\
\hline
\end{tabular}

\section{Conclusions}

Sodium hydroxide can be used to modify the acetate fiber by oxidation. Fixation rate of reactive dyes can be well characterized the changes of acetate fiber, the higher fixation rate, the better modification result. The breaking strength of modified acetate fiber is decreased and the moisture regain is increased. The mass loss rates is directly proportional to the amount of sodium hydroxide.

\section{References}

[1] B P Corban: Textile fibers to Fabrics (Hill book company, 1983)

[2] J L Marjory: Introductory textile science (CBS College Publishing, 1981)

[3] Klemm D, Philipp B, Heinze T, Heinze U, Wagenknecht W.Comprehensive cellulose chemistry, vol. 2. Weinheim: WileyeVCH; 1998.

[4] J. Koh et al. Reactive dyeing properties of novel regenerated cellulosic fibers. Dyes and Pigments , 64(2005), p.9

[5] J. Koh et al. / Dyes and Pigments 64 (2005), p.9. 